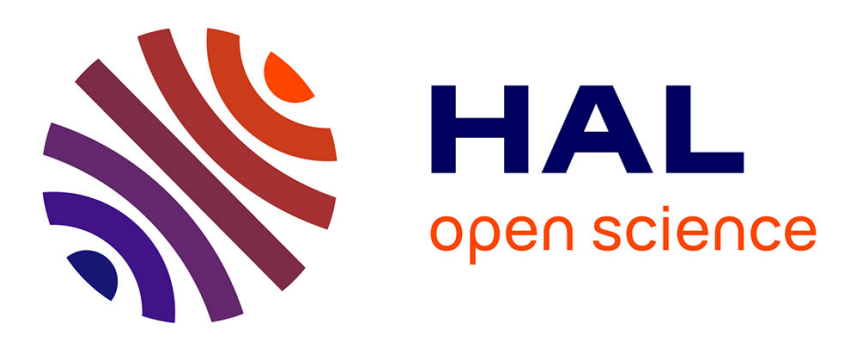

\title{
The analysis of the electric heating of the WC-Co hard-alloy under consideration of the dependence of it's characteristics on the temperature
}

\author{
A. Raichenko, E. Chernikova, E. Olevsky
}

\section{- To cite this version:}

A. Raichenko, E. Chernikova, E. Olevsky. The analysis of the electric heating of the WC-Co hard-alloy under consideration of the dependence of it's characteristics on the temperature. Journal de Physique IV Proceedings, 1993, 03 (C7), pp.C7-1235-C7-1239. 10.1051/jp4:19937190 . jpa-00251823

HAL Id: jpa-00251823

https://hal.science/jpa-00251823

Submitted on 1 Jan 1993

HAL is a multi-disciplinary open access archive for the deposit and dissemination of scientific research documents, whether they are published or not. The documents may come from teaching and research institutions in France or abroad, or from public or private research centers.
L'archive ouverte pluridisciplinaire HAL, est destinée au dépôt et à la diffusion de documents scientifiques de niveau recherche, publiés ou non, émanant des établissements d'enseignement et de recherche français ou étrangers, des laboratoires publics ou privés. 


\title{
The analysis of the electric heating of the WC-Co hard-alloy under consideration of the dependence of it's characteristics on the temperature
}

\author{
A.I. RAICHENKO, E.S. CHERNIKOVA and E.A. OLEVSKY \\ Institute for Problems of Materials Science of Ukrainian Academy of Science, 3 Krzhizhanovsky str., Kiev \\ 252180, Ukraine
}

The mathematical method of the calculation of the temperature in the model: electrode/ punchspecimen-electrode/punch is presented in the given work. The thermal processes taking place in the electrode/punches during passage of electric current through the electrode/punch-specimenelectrode/punch system slightly resemble the thermal processes in electric butt welding of bars. In contrast to electric welding, in electric heating of the porous specimen it is necessary to take into account the thermal processes taking place in the zone between the electrode/punches, namely, in the specimen. Since the electrode/punches are not only in electrical but also in thermal contact with the specimen, the description of the thermal processes in the specimen with no allowance made for the generation and transfer of heat in the electrode/punches is incomplete. The previous studies do not take into account porosity of the treated specimen and the temperature dependence of the thermo- and electrophysical parameters and density. It is well known that in electric current sintering the powder often transforms into a connected medium (porous or pore-free) in a short period of time; there are no specific contact effects in this type of medium. The proposed theory is suitable for describing heating of either such a powder sintered by electric current or of a porous specimen produced by the conventional cold compacting-sintering in furnace method. The aim of this work was to describe mathematically the heat transfer in the electrode/punch-specimen-electrode/punch model in the conditions: the electric current which passes through the system does not change with time, the side surfaces are thermally insulated, and there are no contact effects. The linear dimensions of the model are assumed to be constant. The electrical resistance, heat conduction and others properties are temperature dependent. The dependencies of the WC-Co hard-alloy's characteristics and characteristics of the electrode/punch's materials on the temperature were corrected in the calculations. In this case the heat conduction equations are nonlinear. The analytic solution, which is based on the assumption that the characteristics of the hard-alloy and electrode/punch's material are constant, are examined also in this work. The comparison of the given method with the analytic calculation and the experimental results is shown the advantages of the developed method, taking into consideration temperature dependence of properties. 
In examining heating of porous materials with electric current the heat- and electro- physical processes taking place in the electrode/punches are usually not taken into account (1-5). In certain cases, analysis in its qualitative form is carried out for the processes of removal of heat from the porous material (sintered powder) and with the contacting electrode/punches and the die $(2,6,7)$. In $(8)$, the equation for the temperature in the metal between the electrode was derived taking into account heat transfer in the electrodes but the expression for the temperature field in the electrodes was not given. In using the method of finite elements for calculating (9) the temperature of the powder through which the electric current is passed, and the temperatures of the electrode/punches are assumed to be uniform in space (but do not coincide).

The thermal processes taking place in the electrode/punches in passage of electric current through the electrode/punch-specimen-electrode/punch system slightly resemble the thermal processes in electric butt welding of bars. The authors of $(10)$ examinde the relationships governing heating with the current in resistance butt welding bars of the same cross section, formulated the assumptions which were used as a basis for calculating the process, and showed a method of linearizing the differential equation of heating of the rods with the current which varies with time and with an additional local heat source determined by the current concentration in the near-contact zone. In this work, we examine the types of heating which are limiting, i.e., define the upper and lower limits of the cases of heating encountered in practise in resistance butt welding which are determined by the real conditions of removal of heat from the working sections of the rod. In the first limiting case the rods are assumed to be unlimited and the effect of heat removal to the nonheated ends of the rod and the mass of the current conductors are ignored. In the second case it is assumed that the rods are characterized by the maximum heat removal into the mass of the current conductors which results in the constant temperature of the end cross sections of the working areas.

Electrothermal processes taking place in resistance butt welding were also examined in (11). The authors of (11) developed methods of calculating the temperature distribution along the length of the rods up to the end of heating and the thermal cycle of the welded joint in accordance with the theory of propagation of heat in resistance butt welding; the results of theoretical calculations were verified by means of experiments.

In contrast to electric welding, in electric heating of the porous specimen it is necessary to take into account the thermal processes taking place in the zone between the electrode /punches, and, namely, in the specimen. Since the electrode/punches are not only in electrical but also thermal contact with the specimen, the description of the thermal processes in the specimen with no allowance made for the generation and transfer of heat in the electrode/punches is incomplete. The above studies do not take into account the porosity of the treated specimen and the temperature dependence of the thermo- and electrophysical parameters and density. In the theory of heating of complex electrical conductors (12) the temperature fields which would make it possible to take into account all thermal diffusion processes in the specimen and with the electrode/punches which are in contact with the specimen were not calculated.

It is well known that in electric current sintering the powder often transforms into a connected medium ( porous or pore-free) in a short period of time; there are no specific contact effects in this type of medium. The theory proposed by the authors of this work is suitable for describing heating of either such a powder sintered by electric current or of a porous specimen produced by the conventional cold compacting-sintering in furnace method (12).

The aim of this work was to describe mathematically the heat transfer in the electrode/punch-specimenelectrode/punch model in the conditions: the electric current which passes through the system does not change with time, the side surfaces are thermally insulated, and there are no contact effects. The linear dimensions of the model are assumed to be constant (i.e., in the examined approximation, thermal expansion and shrinkage in the direction of current passage are ignored).Because of the symmetry, the problem can be solved for one half of the model.

If we denote the temperature of the specimen by $T_{1}$ and the temperature of the electrode/punch by $T_{2}$, the process must be governed by the system of defferential equations: 


$$
\begin{aligned}
& \frac{\partial T_{1}}{\partial t}=a_{1} \frac{\partial^{2} T_{1}}{\partial x^{2}}+m_{1} \\
& \frac{\partial T_{2}}{\partial t}=a_{2} \frac{\partial^{2} T_{2}}{\partial x^{2}}+m_{2}
\end{aligned}
$$

where $c_{i}$ is heat capacity; $\gamma_{j}$ is the density of the material; $T_{i}$ is the temperature; $t$ is the time; $\lambda_{i}$ is the heat conductivity coefficient; $x$ is the coordinate counted from the center of the specimen; $r_{i}$ is the specific electrical resistivity; $j$ is the current density (all quantities with $i=1$ relate to the specimen, with $\mathrm{i}=2$ to the electrode/punches; in the range $0 \leq x \leq l_{1}$ the specimen is placed, where as the electrode/punch is placed in the range $l_{1} \leq x \leq l_{1}+l_{2}, \mathrm{a}_{\mathrm{i}}=\lambda_{\mathrm{i}} /\left(\mathrm{c}_{\mathrm{i}} \gamma_{\mathrm{i}}\right)$ is the thermal diffusivity; $\mathrm{m}_{\mathrm{i}}=\left(\mathrm{\rho}_{\mathrm{i}}\right.$ $\left.\mathrm{j}^{2}\right) /\left(\mathrm{c}_{\mathrm{i}} \gamma_{\mathrm{j}}\right)$ is the quantity which can be regarded as the power of the distributed Joule temperature sources.

The problem is solved for the bounary-value conditions

$$
T_{1}(x, 0)=T_{2}(x, 0)=T_{0} \text {; }
$$

$\left.\frac{\partial T}{\partial t}\right|_{x=0}=0$

$T_{2}(l, t)=T_{(7)}$

where $1=1_{1}+l_{2}$. According to Eg.(3), at the initial moment the entire block has a uniform constant temperature; the condition (4) reflects the symmetry of the temperature field, (5) reflects the continuity of the heat flow through the contract between the compact and the electrode/punch, (6) indicates the continuity of the temperature field at this contact, and Eg. (7) reflects the constant temperature at the end of the electrode/punch which is in contact with the current-conducting plate of equipment. The latter condition reflects the effect of the efficient heat removal as a result of a large mass and high heat conductivity of the current-conducting plate. The quantity $c_{i}, g_{i}, r_{i}, l_{i}$ and $j$ are assumed to be constant. However, if the heat removal through the "cold" ends (i.e., the ends which are in contact with the current-conducting plates) of the electrode/punches is not so intensive as to satisfy the condition (7), it is necessary, generally speaking, to connect in the circuit a current-conducting plate, an insulating interlayer, etc. We shall use a different procedure; in evaluating $T(x, t)$ we take into account the actual (experimentally measured) temperature of the "cold" end of the electrode/punch $T_{2}(1, t)=T_{0}$. The experimental dependence of the temperature of the "cold" end of the electrode/punch $\mathrm{T}_{2}(1, t)=\mathrm{T}_{0}$ can be represented analytically in the form $T(1, t)=T_{c}\left(1-e^{-a t}\right)$, where $T_{c}$ and a are constants. Consequently, the accuracy of the heat conductivity equations (1) and (2) must be improved

$$
\begin{aligned}
& \frac{\partial T_{1}}{\partial t}=a_{1} \frac{\partial^{2} T_{1}}{\partial x^{2}}+m_{1}+\alpha T_{c} \ell^{-\alpha t} \\
& \frac{\partial T_{2}}{\partial t}=a_{2} \frac{\partial^{2} T_{2}}{\partial x^{2}}+m_{2}+\alpha T_{c} \ell^{-\alpha t}
\end{aligned}
$$

The solution of the system (8),(9) is written in the explicit form 


$$
\begin{aligned}
& T_{1}(x, t)=T_{c}\left(1-\ell^{-\alpha \ell}\right)+\frac{m_{1} l_{1}^{2}}{2 a_{1}}+\frac{m_{2} l_{2}^{2}}{2 a_{2}}+\frac{m_{1} l_{1} l_{2} c_{1} \gamma_{1}}{a_{2} c_{2} \gamma_{2}}-\frac{m_{1}}{2 a_{1}} x^{2}+2 \frac{l_{1}^{3} c_{1} \gamma_{1} \lambda_{2} m_{2}}{l_{2} \lambda_{1}^{2}}
\end{aligned}
$$

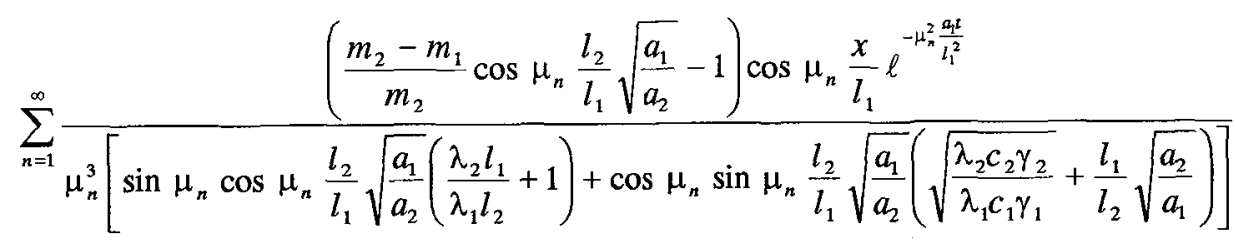

$$
\begin{aligned}
& T_{2}(x, t)=T_{c}\left(1-\ell^{-\alpha t}\right)+\frac{m_{1} c_{1} \gamma_{1} l_{1}\left(l_{1}+l_{2}\right)}{\lambda_{2}}+\frac{m_{2} c_{2} \gamma_{2}\left(l_{2}^{2}-l_{1}^{2}\right)}{2 \lambda_{2}}+\left(m_{2} c_{2} \gamma_{2}-m_{1} c_{1} \gamma_{1}\right) \frac{l_{1} x}{\lambda_{2}}- \\
& \frac{m_{2} c_{2} \gamma_{2} x^{2}}{2 \lambda_{2}}-\frac{2 l_{1}^{2}}{l_{2} a_{1}} \sqrt{\frac{a_{2}}{a_{1}}} m_{2} x^{2} \sum_{n=1}^{\infty}\left[\left(\sqrt{\frac{\lambda_{2} c_{2} \gamma_{2}}{\lambda_{1} c_{1} \gamma_{1}}} \cos \mu_{n} \cos \mu_{n} \sqrt{\frac{a_{1}}{a_{2}}}+\sin \mu_{n} \sin \mu_{n} \sqrt{\frac{a_{1}}{a_{2}}}-\right.\right. \\
& \left.\frac{m_{2}-m_{1}}{m_{2}} \sin \mu_{n} \sin \mu_{n} \frac{l}{l_{1}} \sqrt{\frac{a_{1}}{a_{2}}}\right) \cos \mu_{n} \sqrt{\frac{a_{1}}{a_{2}}} \frac{x}{l_{1}}+\left(\sqrt{\frac{\lambda_{2} c_{2} \gamma_{2}}{\lambda_{1} c_{1} \gamma_{1}}} \cos \mu_{n} \sin \mu_{n} \sqrt{\frac{a_{1}}{a_{2}}}+\right. \\
& \left.\frac{m_{2}-m_{1}}{m_{2}} \sin \mu_{n} \cos \mu_{n} \sqrt{\frac{a_{1}}{a_{2}}} \frac{l}{l_{1}}-\sin \mu_{n} \cos \mu_{n} \sqrt{\left.\frac{a_{1}}{a_{2}}\right)} \sin \mu_{n} \sqrt{\frac{a_{1}}{a_{2}}} \frac{x}{l_{1}}\right]\left\{\mu _ { n } ^ { 3 } \left[\left(\frac{\lambda_{2} l_{1}}{\lambda_{1} l_{2}}+1\right) \sin \mu_{n}\right.\right. \\
& \left.\cos \mu_{n} \sqrt{\frac{a_{1}}{a_{2}}} \frac{l_{2}}{l_{1}}+\left(\sqrt{\frac{\lambda_{2} c_{2} \gamma_{2}}{\lambda_{1} c_{1} \gamma_{1}}}+\sqrt{\frac{a_{2}}{a_{1}} \frac{l_{1}}{l_{2}}}\right) \cos \mu_{n} \sin \mu_{n} \sqrt{\frac{a_{1}}{a_{2}}} \frac{l_{2}}{l_{1}}\right\}^{-1} \ell^{-\mu_{n}^{2} \frac{a_{l} l}{l_{1}^{2}}}
\end{aligned}
$$

where $\mu_{\mathrm{n}}$ are the roots of Eq.(11).

$$
\sqrt{\frac{\lambda_{2} c_{2} \gamma_{2}}{\lambda_{1} c_{1} \gamma_{1}}} \cos \mu_{n} \cos \left(\mu_{n} \frac{l_{2}}{l_{1}} \sqrt{\frac{a_{1}}{a_{2}}}\right)-\sin \mu_{n} \sin \left(\mu_{n} \frac{l_{2}}{l_{1}} \sqrt{\frac{a_{1}}{a_{2}}}\right)=0
$$

We can calculate the dependences showing the extent to which the temperature $\Theta_{1}, \Theta_{2}$ is higher than the temperature $T_{0}$, on the spatial coordinate (for any time $t$ ) on the "bases' $T_{2}(1, t)$, i.e., temperature of the "cold" end of he electrode/punch. The calculated temperature is equal to $T_{2}(1, t)+\Theta_{1}$ The temperature field in the model: the electrode/punch-porous sintered specimen-electrode/punch, may be obtained by the set of the two differential heat conduction equations. The analytic solution, which is based on the assumption that the characteristics of the hard-alloy and electrode/punch's material are constant, are examined, above in this work.

However, the electrical resistance, heat conduction and others properties are temperature dependent. The dependencies of the WC-Co hard-alloy's characteristics and characteristics of the electrode/punch's materials on the temperature were corrected in the calculations. In this case the heat conduction equations are nonlinear, that's why the finite difference approximation in the form of the explicit circut is used for the calculation of these equations (12).

$$
\frac{T_{k}^{v}-T_{k}^{v-1}}{\tau}-a_{k}^{v} \frac{T_{k-1}^{v-1}-2 T_{k}^{v-1}+T_{k+1}^{v-1}}{h^{2}}=m_{k}^{v-1},(
$$


where $T$ is the temperature; $a_{k}{ }^{n}$ is the thermal diffusivity; $m_{k}{ }^{n-1}$ is the power of the distributed Joule's sources; $t, h$ are spacings of the time and coordinate correspondingly; $k=0, n ; n=0, m$ are indexes of the coordinate and time divisions correspondingly.

The boundary condition in the centre of symmetry of the specimen is as the equation (13)

$$
T_{0}{ }^{v}=\left[m_{0}^{\nu-1}+2 a_{1} \frac{T_{1}^{v-1}-T_{0}{ }^{v-1}}{h^{2}}\right] \tau+T_{0}{ }^{v-1}
$$

The boundary condition of the free end, coincided with the condition (7) is as the equation (14)

$$
T_{n}{ }^{\nu}=T_{c}\left(1-e^{-\alpha v \tau}\right)+20
$$

where $T_{c}$ and a are constants.

The continuity of the heat flow through the contact between the specimen and electrode/punch may be represented by formula (15)

$$
\lambda_{1 p}^{v}\left[T_{p}^{v}-T_{p+1}^{\vee v}\right]=\lambda_{2 p}^{v}\left[T_{p-1}-T_{p}\right],(20)
$$

where $1_{1,2}$ are the heat conductions of the hard alloy and electrode/punch correspondingly, $\mathrm{p}$ is an index, corresponding to the boundarys' coordinate between the specimen and electrode/punch. The equation (15) is the discrete analoque to the equation (5).

The program on the algorithmic "BASIC" language was developed to solve the above-mentioned set of the heat conduction equations using the given finite difference circut. This program was adapted to employ one on the PVM ES-1840. The pack of the programs on the "BASIC' language was developed too in order to perform the parabolic regression analysis of the reference data of the WC-Co hardalloy's characteristics and 20KhN3A steel's characteristics.

The two variants of the numerical solution of the formulated problem were considered: current density is $\mathrm{j}=42910^{4} \mathrm{~A} / \mathrm{m}^{2}$ and $78410^{4} \mathrm{~A} / \mathrm{m}^{2}$.

Conclusions.

The mathematical method of the calculation of the pattern's temperature in the model:the electrode/punch-specimen-electrode/punch, are presented in the given work. The dependencies of the WC-Co hard-alloy's characteristics and characteristics of the electrode-punch's materials on the temperature were corrected in the calculations. The analitic solution, which based on the assumption that the characteristics of the hard-alloy and electrode/punch's material are constant, are examined also in this work. The comparision of the given method with the analytic calculation and the experimental results is shown the advantages of the developed method.

\section{References}

[1].M.I.Sericov, A.A.Sletcov and V.M.Umrikhin,Powder.Metall.,(1978),N.12,p.13-17.

[2].T.Sakai,M.Itabasi, and D.Hara,Seysan Kenko,(1966),18,N.2,p.35-40.

[3].S.A.Balankin, S.S.Bashlykov,L.P.Gorbachev,Fiz.-Khim.Obrab.Mater.,(1984),N.2,p. 124-129.

[4].A.I.Raichenko,G.L.Burenkov, A.F.Khrienko,Powder Metall.,(1976),N8,p.25-30.

[5].V.A.Belyi,V.V.Meshkov, N.K.Myshkin,Docl., Acad.Nauk SSSR,(1982), v.265,N5,p.1121-1124.

[6].V.V.Meshkov, N.K.Myshkin,A.I.Sviridenok, Powder Metall.,(1984),N3,p.36-39.

[7].V.K.Yaroshevich,Ya.S.Genkin,V.A.Vereshchagin, Electric Resistance Hardening,Nauka i Tekhnika, Mimsk (1982)

[8].A.V.Pilipchenko,A.I.Tsitrin, A.N.Khomchenko,Powder.Metall.,(1987),N.3,p26-29.

[9].F.W.Lenel, J.Met.,(1955),v.7,N1,p.158-167.

[10].V.I.Tumanov,Properties of alloys of the tungsten carbide-cobalt system,Metallutgiya,Moscow (1971).

[11].I.K.Kikoin,Tables of Physical Quantities,Atomizdat,Moscow,(1976).

[12].A.I.Raichenko,E.S.Chernikova,Powder Metall., (1989), V.28,N7,p.319-325. 